\title{
Gravity's immunity from vacuum: the holographic structure of semiclassical action
}

\section{T. Padmanabhan}

Published online: 26 September 2006

(C) Springer Science+Business Media, LLC 2006

\begin{abstract}
Principle of equivalence, general covariance and the demand that the variation of the action functional should be well defined, lead to a generic Lagrangian for semiclassical gravity of the form $L=Q_{a}{ }^{b c d} R_{b c d}^{a}$ with $\nabla_{b} Q_{a}{ }^{b c d}=0$. The expansion of $Q_{a}^{b c d}$ in terms of the derivatives of the metric tensor determines the structure of the theory uniquely. The zeroth order term gives the Einstein-Hilbert action and the first order correction is given by the Gauss-Bonnet action. Remarkably, any such Lagrangian can be decomposed into a surface and bulk terms which are related holographically. The equations of motion can be obtained purely from a surface term in the gravity sector and hence gravity does not respond to the changes in the bulk vacuum energy density.
\end{abstract}

\section{Gravity: kinematics versus dynamics}

The elegance of general relativistic description of gravity rests on the geometric structure, which - in turn - is based on the principle of equivalence (PE). In its simplest form, PE allows the description of gravity in terms of a metric tensor and determines the kinematics of gravity ('how gravity tells matter to move') by invoking special relativity in the local inertial frames.

Contrast this with the description of dynamics of gravity ('how matter tells spacetime to curve') for which we completely lack a similar guiding principle. Classical dynamics has to arise from semiclassical limit of quantum gravity

Third award in the 2006 Essay Competition of the Gravity Research Foundation.

T. Padmanabhan $(\bowtie)$

IUCAA, Pune University Campus, Ganeshkhind, Pune 411 007, India

e-mail: nabhan@iucaa.ernet.in 
through the variation of the semiclassical action functional. But we lack a guiding principle to choose such an action functional! There are several serious issues which crop up when we try to determine the action functional:

- If $g_{a b}$ are the dynamical variables, the natural action functional should be quadratic in the derivatives $\partial g$ of $g_{a b}$. But PE - which allows us to reduce $g_{a b} \rightarrow \eta_{a b}, \partial_{c} g_{a b} \rightarrow 0$ in any local region - prevents the existence of such a generally covariant action. So, general covariance, combined with PE, forces us to include $\partial^{2} g$ terms in the action; but then the variational principle becomes ill-defined and needs a special treatment. This situation is unparalleled in any other theory in physics (like e.g Yang-Mills).

- Further, any such action can only provide a low energy effective description of gravity. The semiclassical theory is likely to exist in some $D$ dimensional spacetime with $D>4$ and quantum corrections will add higher order correction terms involving squares of the curvature etc. We have no guiding principle or symmetry to determine these higher order terms.

A closely related question is: What are the true degrees of freedom of gravity? The description in terms of $g_{a b}$ may be most geometrical but it is highly gauge redundant. Any description in terms of an alternative set of variables has important conceptual implications for dynamics - especially for the problem of the cosmological constant. For example, if the degrees of freedom of gravity in a spatial volume $V$ scale as the area $S$ of the bounding surface rather than the volume, then the bulk cosmological constant cannot produce gravitational effects. The reduction from volume to area changes the energy density of the vacuum that is coupled to gravity from the gigantic $L_{P}^{-4}$ to the observed value $L_{P}^{-4}\left(L_{P}^{2} / S\right)$ with $S \approx H^{-2}$, as shown in Refs. [1,2].

I will now provide a paradigm in which all these issues can be successfully tackled at one go. In particular I will show that theories which obey principle of equivalence and general covariance have a generic structure for their low energy action functional, using which one can systematically obtain corrections to classical gravity. When the action is expanded in the powers of curvature, one obtains the Einstein-Hilbert (EH) action as the unique zero-order term along with Gauss-Bonnet (GB) type correction as the unique first-order term. What is probably even more remarkable is that all these action functionals allow a natural, holographically dual, description; that is, the action functional can be expressed as a sum of bulk and surface terms with a definite relationship between the two. The gauge redundancy of geometric description therefore allows all these theories to be described entirely in terms of surface degrees of freedom thereby suggesting a natural solution to the cosmological constant problem.

\section{Structure of gravitational actions}

Consider a (generalized) theory of gravity in D-dimensions based on a generally covariant scalar Lagrangian $L$ which is a functional of the metric $g^{a b}$ 
and curvature $R_{b c d}^{a}$. Instead of treating $\left[g^{a b}, \partial_{c} g^{a b}, \partial_{d} \partial_{c} g^{a b}\right]$ as the independent variables, it is convenient to use $\left[g^{a b}, \Gamma_{k l}^{i}, R^{a}{ }_{b c d}\right]$ as the independent variables. The curvature tensor $R_{b c d}^{a}$ can be expressed entirely in terms of $\Gamma_{k l}^{i}$ and $\partial_{j} \Gamma_{k l}^{i}$ and is independent of $g^{a b}$. To investigate the general "off-shell" structure of the theory, let us note that any scalar which depends on $R^{a}{ }_{b c d}$ can be written in the form $L=Q_{a}^{b c d} R_{b c d}^{a}$ with the tensor $Q_{a}^{b c d}$ depending on curvature and metric. Varying the action functional will give, quite generically,

$$
\delta A=\delta \int_{\mathcal{V}} d^{D} x \sqrt{-g} L=\int_{\mathcal{V}} d^{D} x \sqrt{-g} E_{a b} \delta g^{a b}+\int_{\mathcal{V}} d^{D} x \sqrt{-g} \nabla_{j} \delta \nu^{j},
$$

where $\delta v^{j}$ depends on $\left(\delta \sqrt{-g} L / \delta g^{a b}\right)$ and $\left(\delta L / \delta R^{a}{ }_{b c d}\right)$. To have a good variational principle leading to the result $E_{a b}=T_{a b}$, we need to assume that $n_{a} \delta v^{a}=0$ on $\partial \mathcal{V}$ where $n_{a}$ is the normal to the boundary. In general this requires a particular combination of the variations in the "coordinates" $\left[\delta g_{a b}\right]$ and the "momenta" $\left[\nabla_{c} \delta g_{a b}\right]$ to vanish and we need to put conditions on both the dynamical variables and their derivatives on the boundary. It is more reasonable in a quantum theory to choose either the variations of coordinates or those of momenta to vanish rather than a linear combination. It can be shown [1] that this requires the condition $\nabla_{c} Q^{i j c d}=0$ provided $Q^{i j c d}$ has a Taylor series expansion in the curvature tensor. Because of the symmetries, this implies that $Q^{a b c d}$ is divergence-free in all indices.

Using the antisymmetry of $Q_{a}{ }^{b c d}$ in $c, d$ we can write our Lagrangian as:

$$
\sqrt{-g} L=\sqrt{-g} Q_{a}^{b c d} R_{b c d}^{a}=2 \sqrt{-g} Q_{a}^{b c d}\left[\partial_{c} \Gamma_{d b}^{a}+\Gamma_{c k}^{a} \Gamma_{d b}^{k}\right]
$$

An integration by parts followed by straightforward algebra (see Sect. 5 of Ref. [1] for details) now gives

$$
\sqrt{-g} L=2 \partial_{c}\left[\sqrt{-g} Q_{a}^{b c d} \Gamma_{b d}^{a}\right]+2 \sqrt{-g} Q_{a}^{b c d} \Gamma_{d k}^{a} \Gamma_{b c}^{k} \equiv L_{\text {sur }}+L_{\text {bulk }}
$$

This result shows that any such gravitational Lagrangian, built from metric and curvature, has a separation into a surface term (first term) and bulk term (second term) in a natural but non covariant manner. Ignoring the surface term, one can obtain the same covariant equations of motion $E_{a b}=T_{a b}$ even from a non covariant Lagrangian.

We note that Eq. (3) represents the most general effective Lagrangian for gravity which is consistent with principle of equivalence, general covariance and the dynamical requirement that a well-defined variational principle should exist. The structure of the theory is specified by a single divergence-free fourth rank tensor $Q_{a}^{b c d}$ having the symmetries of the curvature tensor. The semi classical, low energy, action for gravity can now be determined from the derivative expansion of $Q_{a}{ }^{b c d}$ in powers of number of derivatives: 


$$
Q_{a}{ }^{b c d}(g, R)=\stackrel{(0)}{Q}_{a}^{b c d}(g)+\alpha \stackrel{(1)}{Q}_{a}^{b c d}(g, R)+\beta \stackrel{(2)}{Q}_{a}^{b c d}(g, R, \nabla R)+\cdots
$$

where $\alpha, \beta, \cdots$ are coupling constants. To determine the first term, say, we only need to obtain all the possible fourth rank tensors $Q^{a b c d}$ which (i) have the symmetries of curvature tensor; (b) are divergence-free and (iii) are made from $g^{a b}$; similarly, to obtain the next term, we allow the tensor $Q^{a b c d}$ to depend on $g^{a b}$ and $R_{b c d}^{a}$ etc. Interestingly enough, at the first two orders, this leads to all the gravitational theories (in D dimensions) in which the field equations are no higher than second degree, though we did not demand that explicitly.

To see this, let us note that if we do not use the curvature tensor, then we have just one unique choice for zeroth order, made from metric:

$$
\stackrel{(0)}{Q}_{a}^{b c d}=\frac{1}{2}\left(\delta_{a}^{c} g^{b d}-\delta_{a}^{d} g^{b c}\right),
$$

which satisfies our constraints. When $Q_{a}{ }^{b c d}$ is built from metric alone, Eq. (3) becomes

$$
\sqrt{-g} L=\partial_{c}\left[\sqrt{-g}\left(g^{b d} \Gamma_{b d}^{c}-g^{b c} \Gamma_{b a}^{a}\right)\right]+\sqrt{-g}\left(g^{b d} \Gamma_{d j}^{a} \Gamma_{b a}^{j}-g^{b c} \Gamma_{a j}^{a} \Gamma_{b c}^{j}\right)
$$

which is precisely the bulk-surface decomposition for Einstein-Hilbert action.

Next, if we allow for $Q_{a}{ }^{b c d}$ to depend linearly on curvature, then we have the following additional choice of tensor with required symmetries:

$$
\stackrel{(1)}{Q}_{a b c d}=R_{a b c d}-G_{a c} g_{b d}+G_{b c} g_{a d}+R_{a d} g_{b c}-R_{b d} g_{a c} .
$$

(In four dimensions, this tensor is essentially the double-dual of $R_{a b c d}$ and in any dimension can be obtained from $R_{a b c d}$ using the alternating tensor [3].) In this case, we get

$$
\begin{aligned}
L & =\frac{1}{2}\left(g_{i a} g^{b j} g^{c k} g^{d l}-4 g_{i a} g^{b d} g^{c k} g^{j l}+\delta_{a}^{c} \delta_{i}^{k} g^{b d} g^{j l}\right) R_{j k l}^{i} R_{b c d}^{a} \\
& =\frac{1}{2}\left[R^{a b c d} R_{a b c d}-4 R^{a b} R_{a b}+R^{2}\right] .
\end{aligned}
$$

This is just the Gauss-Bonnet (GB) action which is a pure divergence in 4 dimensions but not in higher dimensions. The unified procedure for deriving Einstein-Hilbert action and GB action shows that they are more closely related to each other than previously suspected. The fact [4] that string theoretical models get $G B$ type terms as corrections is noteworthy in this regard. We can similarly determine the higher order corrections.

We shall now show that there is another striking relation between the surface and bulk terms in the Lagrangian in Eq. (3). To see this we begin by noting that, 
the $L_{\text {bulk }}=2 \sqrt{-g} Q_{a}^{b c d} \Gamma_{d k}^{a} \Gamma_{b c}^{k}$ and $L_{\mathrm{sur}}=2 \partial_{c}\left[\sqrt{-g} Q_{a}^{b c d} \Gamma_{b d}^{a}\right]$, are always related by

$$
L_{\text {sur }}=-\partial_{c}\left(\delta_{b}^{k} \frac{\partial L_{\mathrm{bulk}}}{\partial \Gamma_{c b}^{k}}\right) .
$$

Using $\partial_{c} g_{a b}=\Gamma_{a b c}+\Gamma_{b a c}=\Gamma_{j k}^{l}\left[g_{l a} \delta_{b}^{j} \delta_{c}^{k}+g_{l b} \delta_{a}^{j} \delta_{c}^{k}\right]$ we can express partial derivatives with respect $\Gamma_{j k}^{l}$ in terms of those with respect to $\partial_{c} g_{a b}$. This leads to the the remarkable result that, for a generic action functional in Eq. (3) the bulk and surface terms are related by

$$
L_{\mathrm{sur}} \propto \partial_{a}\left(g_{b c} \frac{\delta L_{\mathrm{bulk}}}{\delta\left(\partial_{a} g_{b c}\right)}\right) .
$$

This makes the action intrinsically holographic with the surface term containing an equivalent information as the bulk. It has been shown earlier (see Refs. [5,6]) that the bulk and surface terms of Einstein-Hilbert action in Eq. (6) are related by this identity. The current result shows that this is very general and is based only on the principle of equivalence (which allows the gravity to be described by a metric), general covariance (which fixes the generic form of the action) and demand for the existence of a well defined variational principle (which requires $\nabla_{a} Q^{a b c d}=0$ ).

Further, just as in the case of EH action [7], it is possible to reformulate the theory retaining only the surface term for the gravity sector. If we consider an action principle with based on $\left(A_{m}+A_{s}\right)$ where $A_{m}$ is the matter action and $A_{s}$ is the action obtained from $-L_{\text {sur }}$ (the minus sign is just to ensure that this is the term which, when added to our action will cancel the surface term) then, for variations that arise from displacement of a horizon normal to itself, one gets the equation $\left(E_{a b}-\frac{1}{2} T_{a b}\right) \xi^{b} \xi^{a}=0$ where $\xi^{a}$ is a null vector. Combined with the (generalised Bianchi) identity $\nabla_{a} E^{a b}=0$ this will lead to standard field equations with a cosmological term $E_{a b}=(1 / 2) T_{a b}+\Lambda g_{a b}$ just as in the case of Einstein-Hilbert action (derived by this route in Ref. [7]).

In this approach, which uses only surface degrees of freedom for gravity, the basic field equations are $\left(E_{a b}-\frac{1}{2} T_{a b}\right) \xi^{b} \xi^{a}=0$ where $\xi^{a}$ is a $n u l l$ vector. So the addition of a cosmological constant - by the change $T_{a b} \rightarrow T_{a b}+\Lambda g_{a b}$-leaves the equations invariant. Gravity ignores the bulk vacuum energy density! When generalised Bianchi identity is used, the cosmological constant does arise as an integration constant; but now, it can be set to any value as a feature of the solution to the field equations. (It has a status similar to mass in the Schwarzschild metric). This provides a basic reason for ignoring the bulk and its changes during various phase transitions in the universe. The thermodynamic paradigm also impies that if an observer has a horizon, we should work with the degrees of freedom confined by the horizon. This changes the pattern of vacuum fluctuations and leads to the correct, observed value of the cosmological constant [2].These features arise purely from principle of equivalence and general covariance and is not specific to Einstein's theory. Any theory of gravity described by 
a metric will have similar features and hence higher order quantum gravitational corrections are likely to respect these principles.

\section{Conclusions}

Let us summarize what has been achieved in this formalism and how it successfully addresses the questions raised in the Sect. 1.

Principle of equivalence, general covariance and the demand for the existence of a well-defined variational principle requires the Lagrangian to be of the form $L=Q_{a}{ }^{b c d} R^{a}{ }_{b c d}$ with $\nabla_{b} Q_{a}{ }^{b c d}=0$. The low energy effective action for gravity is then determined by the derivative expansion of $Q_{a}{ }^{b c d}$ in powers of number of derivatives, given by Eq. (4). The first term gives Einstein-Hilbert action and the second one is Gauss-Bonnet action. It is worth recalling that such a Gauss-Bonnet term arises as the correction in string theories [4], as to be expected from our general principle.

It is remarkable that any such Lagrangian $L=Q_{a}{ }^{b c d} R^{a}{ }_{b c d}$ with $\nabla_{b} Q_{a}{ }^{b c d}=0$ can be decomposed into a surface and bulk terms which are related holographically. The equations of motion can be given a thermodynamic interpretation [5,6] expressible as $T \mathrm{~d} S=\mathrm{d} E+P \mathrm{~d} V$ with the surface term interpreted as entropy. This fact, that any theory based on principle of equivalence and general covariance can be described by an action principle involving only the surface degrees of freedom, cuts right into the heart of the matter. The equations of motion are invariant under the changes to the vacuum energy $T_{a b} \rightarrow T_{a b}+\Lambda g_{a b}$ and we have a natural solution to the cosmological constant problem. Note that, this approach, unlike many others, can handle the changes to the vacuum energy density arising due to phase transitions in the early universe. The observed arises due to the vacuum fluctuations in a region confined by the horizon and-in that sense-is coupled to the surface degrees of freedom of gravity.

Finally, note that the thermodynamic interpretation (which is on-shell) as well as the holographic description (which is off-shell) are also applicable to quantum corrections to the Einstein-Hilbert Lagrangian. This provides a general principle for determining the correction terms and constraining the structure of underlying theory.

\section{References}

1. Padmanabhan, T.: Dark energy: mystery of the millennium [astro-ph/0603114]

2. Padmanabhan, T.: Class. Quan. Grav. 22, L107-L110 (2005) [hep-th/0406060]

3. Lovelock, D.: J. Math. Phys. 12, 498 (1971)

4. Zwiebach, B.: Phys. Lett. B 156, 315 (1985)

5. Padmanabhan, T.: Class. Quan. Grav. 21, L1 (2004) [gr-qc/0310027]

6. Padmanabhan, T:: Phys. Rep. 406, 49 (2005) [gr-qc/0311036]

7. Padmanabhan, T.: Int. J. Mod. Phys. D 14, 2263-2270 (2005) [gr-qc/0510015]; [gr-qc/0412068] 